\title{
Observations of Superluminous Supernovae and their Host Galaxies
}

\author{
Giorgos Leloudas ${ }^{1,2}$ \\ ${ }^{1}$ Department of Particle Physics and Astrophysics, Weizmann Institute of Science, \\ Rehovot 7610001, Israel \\ ${ }^{2}$ Dark Cosmology Centre, Niels Bohr Institute, University of Copenhagen, \\ Juliane Maries vej 30, 2100 Copenhagen, Denmark
}

\begin{abstract}
Superluminous supernovae (SLSNe) were discovered during the last decade by widefield surveys. Despite their importance for stellar evolution and mass loss, especially at low metallicities, and their utility as high-redshift probes and possible distance indicators, their nature remains poorly understood. I present here new exciting observations of SLSNe and their host galaxies that offer new clues in the hunt for their progenitors and explosion mechanism. These include constraints on the ages and the metallicities at the SN environments, double peaks revealed by early observations, the presence of $\mathrm{H}$ in nebular spectra, and the first evidence on the explosion geometry, through polarimetry.
\end{abstract}

Keywords. supernovae: general, galaxies: dwarf

About 10 years after the discovery of SLSNe, we are entering now the era of large samples, where significant numbers can be studied in a collective manner. For example, Nicholl et al. (2015a) collected and analysed the optical light curves and spectra of 24 SLSNe I/R, showing that the data is consistent with explosions ejecting large amounts of material (3-30 $\left.\mathrm{M}_{\odot}\right)$. Both magnetar and CSM models, despite their shortcomings, provide acceptable solutions to the data, showing that the explosion mechanism and source of extreme luminosity of these events still remain a mystery. Here, I present additional constraints on the nature of SLSNe. I focus on (i) the study of the SLSN host galaxies and (ii) new insights from new, often surprising, observables.

\section{SUSHIES}

SUSHIES is the acronym for the survey of SUperluminous Supernova Host galaxIES that we have been conducting for the past years. Our purpose is to collect multiwavelength imaging and spectroscopy (when feasible) of the hosts of all SLSNe that have been publicly announced. Our first publication, focused on the spectroscopy of SLSN hosts (Leloudas et al. 2015a). In this work, we observed the following:

(a) H-poor SLSNe (I/R) frequently $(\sim 50 \%)$ occur in galaxies with remarkably strong emission lines $(\mathrm{EW}>100 \AA$ in $[\mathrm{O}$ III] $)$. This cannot be a chance coincidence as extreme emission line galaxies are rare. The SLSN I/R hosts are dwarf galaxies with median values of $10^{8.0} M_{\odot}$ for the stellar mass, $0.27 Z_{\odot}$ for the metallicity, and $10^{-8.8} M_{\odot} \mathrm{yr}^{-1}$ for the specific star formation rate.

(b) H-rich SLSNe II (IIn) typically occur in different environments than H-poor SLSNe: more massive, more metal-rich and with softer radiation fields.

These facts led us to the associated conclusions:

- SLSNe I/R come from a very young stellar population. They are found in environments that are even younger (on average) than those of GRBs and they probably 
represent the first stellar explosions in a starburst. This implies that they have very massive progenitors.

- The difference between the SLSN I/R and SLSN II environments suggests that the progenitors of these systems are really different. It is unlikely that SLSNe II are just SLSNe I surrounded by some additional H-rich CSM.

Our conclusions were confirmed in the detailed study of the most nearby H-poor SLSN host. In Thöne et al. (2015), we derived limits for the age of the stellar population at the location of PTF12dam using various methods. The most constraining value we obtained was down to $3 \mathrm{Myr}$, implying a progenitor that could easily exceed $60 M_{\odot}$. The SUSHIES survey is ongoing.

\section{New clues and insights}

Besides the evolution of light curves and spectra, observations of SLSNe have yielded a number of important observables. These include:

- Early peaks. Leloudas et al. (2012) showed that the rise of SN 2006oz was not smooth but that it showed an initial peak in individual colour light curves and a plateauish behavior in the bolometric light curve. This finding has now been confirmed by Nicholl et al. (2015b) in observations of LSQ14bdq, demonstrating that double-peaked light curves are common in SLSNe.

- Nebular spectroscopy. Despite their high luminosity, only a handful of nebular spectra of SLSNe have been obtained for the most nearby events. In Yan et al. (2015), we presented the surprising discovery that the nebular spectrum of PTF13ehe, which was $\mathrm{H}$-poor at early times, showed an $\mathrm{H} \alpha$ line $(\mathrm{FWHM} \sim 4500 \mathrm{~km} / \mathrm{s})$ at +300 days. We interpreted this finding within the pulsational pair-instability model, but other explanations are possible.

- Geometrical constraints. Polarimetry is a powerful tool to study the geometry of unresolved sources such as SN explosions. In Leloudas et al. (2015b), we presented the first polarimetric observations of a SLSN I. During our campaign (from -8 to +17 days in the rest frame), LSQ14mo did not show any significant polarisation or any statistically significant variation in the measured Stokes parameters. Our conclusion is that LSQ14mo did not present important deviations from spherical symmetry during these phases. We are planning to build a meaningful sample of SLSNe with polarimetry in order to determine whether the LSQ14mo low levels of polarisation are a characteristic of the entire class and study the associated implications for the proposed explosion models.

Any model wishing to explain SLSNe, should be able to successfully accommodate all these observables in addition to the light curve and spectral evolution and the environmental constraints.

\section{References}

Leloudas, G. et al. 2012, A\& A, 541, 129

Leloudas, G. et al. 2015a, MNRAS, 449, 917

Leloudas, G. et al. 2015b, ApJL, in press

Nicholl, M. et al. 2015a, MNRAS, 452, 3869

Nicholl, M. et al. 2015b, ApJ, 807, 18

Thöne, C. C. et al. 2015, MNRAS, 451, 65

Yan, L. et al. 2015, ApJ, in press, arXiv: 1508.04420 